\title{
Structure of a linear array of hollow vortices of finite cross-section
}

\author{
By G. R. BAKER, P. G. SAFFMAN AND J. S. SHEFFIELD \\ Applied Mathematics, California Institute of Technology, Pasadena
}

(Received 2 September 1975)

Free-streamline theory is employed to construct an exact steady solution for a linear array of hollow, or stagnant cored, vortices in an inviscid incompressible fluid. If each vortex has area $A$ and the separation is $L$, there are two possible shapes if $A^{\frac{1}{2}} / L$ is less than a critical value 0.38 and none if it is larger. The stability of the shapes to two-dimensional, periodic and symmetric disturbances is considered for hollow vortices. The more deformed of the two possible shapes is found to be unstable while the less deformed shape is stable.

\section{Introduction}

The recent observations by Brown \& Roshko (1974) of organized vortex structures in the turbulent mixing layer have rekindled interest in the hydrodynamics of arrays of parallel line vortices. Moore \& Saffman (1975) argued that the spacing of the vortex structures was controlled by the fact that there is an upper limit on the line density of a linear array of vortices of finite cross-section in non-viscous incompressible flow. When the vortices come too close, the induced straining fields are too intense for the individual vortices to exist in a steady state. However, they restricted their analysis to uniform vortices with constant vorticity in the cores, and the critical density or spacing was determined by an approximate argument (which was however supported by numerical work) because exact analysis was too hard.

It turns out that if the vortex cores are hollow or stagnant, so that the vorticity is concentrated into vortex sheets on the surfaces of the vortices, then the problem can be solved exactly by the free-streamline theory of inviscid, incompressible, two-dimensional flow, and the purpose of this paper is to present the calculation as a contribution to the theory of vortices. We see no direct physical application of the results, but similar calculations for two-dimensional arrays may be of interest in the theory of uniformly rotating superfluid helium, and the exact results provide a means of checking the approximate argument of Moore \& Saffman. A similar calculation was carried out (before the present work was done) by Hill (1975) for a single hollow vortex in a uniform straining field. 


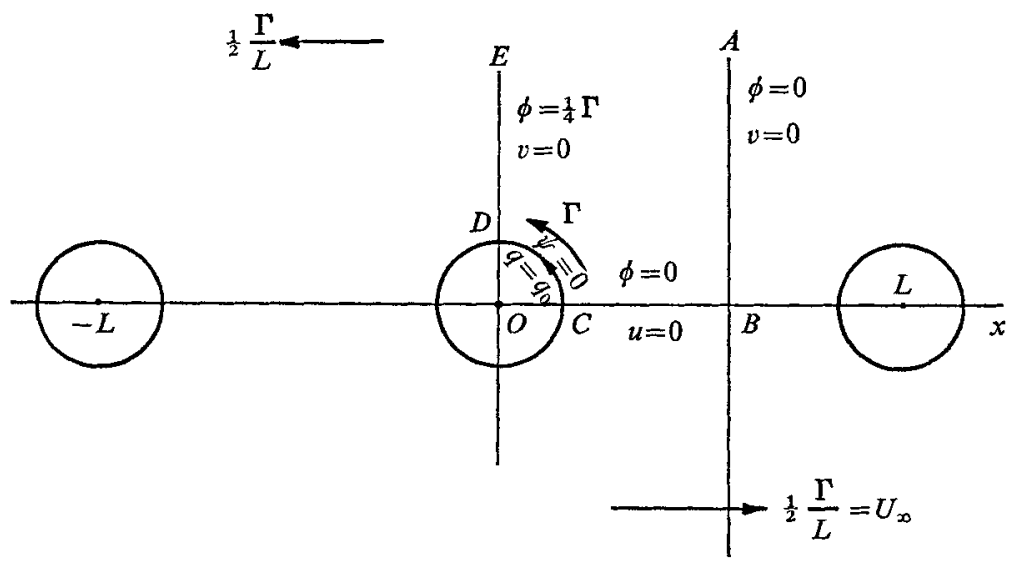

FIGURE 1. The physical plane for a regular array of vortices with fore-and-aft symmetry.

\section{The physical plane}

We consider an infinite linear array of identical vortices lying on the $x$ axis with centres at $n L, n=0, \pm 1, \pm 2, \ldots$. Each vortex is hollow or has a stagnant core. In steady flow, constant pressure inside the cores requires that the fluid speed has a constant value, $q_{0}$ say, on the boundary of each vortex. The circulation $\Gamma$ about each vortex is related to $q_{0}$ by

$$
\Gamma=P q_{0},
$$

where $P$ is the perimeter of each vortex.

At large distances, the array looks like a vortex sheet of strength $2 U_{\infty}$, where

$$
U_{\infty}=\frac{1}{2} \Gamma / L \text {. }
$$

The array is characterized by the dimensionless ratio

$$
R=U_{\infty} / q_{0}=\frac{1}{2} P / L
$$

We shall calculate a unique steady solution for $0<R<1$ in which each vortex has fore-and-aft symmetry, i.e. is symmetrical about the $x$ axis and the line parallel to the $y$ axis through its centre. It can be shown (see appendix) that no solutions with this symmetry exist for $R>1$ and that reflexional symmetry about the centre implies fore-and-aft symmetry.

The limit $R=0$ corresponds to an array of point vortices or a single vortex in unbounded fluid, according as the limit is reached by $P \rightarrow 0$ or $L \rightarrow \infty . R=1$ gives a vortex sheet in which each vortex is pulled out longitudinally and squeezed sideways to lie along a length $L$ of the $x$ axis. Notice that in the limit $R=1$ and the limit $P=0$ the area $A$ of each vortex is zero.

The deformation of the cores is conveniently measured by $P / A^{1}$, which has the minimum value of $2 \pi^{\frac{1}{2}}$ for a circle and becomes large with the eccentricity. Weare interested in how the deformation depends upon the spacing for vortices of given size and strength. The area $A$ is a more basic measure of the size than the peri- 

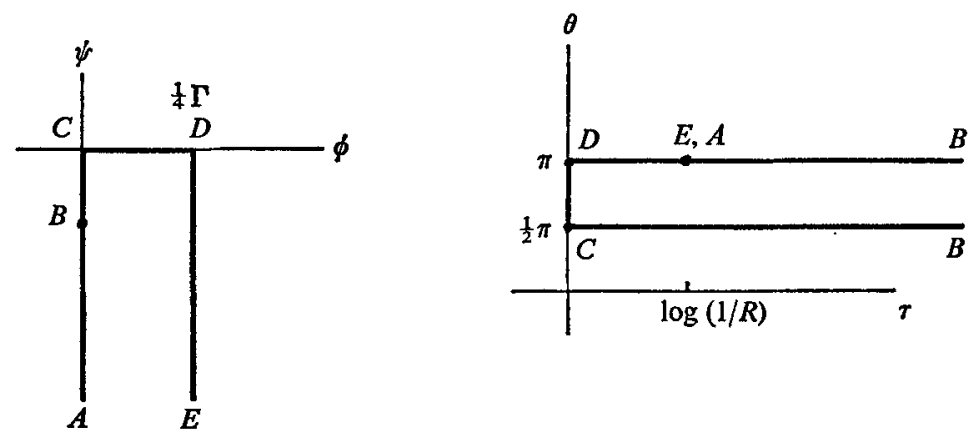

Fraure 2. The mappings of the contour $A B C D E$ in the physical plane into the potential $(\phi, \psi)$ plane and hodograph $(\tau, \theta)$ plane.

meter and $A^{\frac{1}{2}} / L$ is a dimensionless quantity that specifies the relative spacing of the array. The procedure is to calculate $P / A^{\frac{1}{2}}$ and $A^{\frac{1}{2}} / L$ as functions of $R$, and by eliminating $R$ obtain the deformation in terms of the spacing.

The physical plane is shown in figure 1. Because of the symmetry it suffices to calculate the flow inside the contour $A B C D E$. Either the direction or magnitude of the velocity is known on the contour, and the methods of free-streamline theory can therefore be applied by mapping the potential plane into the hodograph plane.

\section{The mappings}

We introduce the complex variable $z=x+i y$, the complex potential $w=\phi+i \psi$, the complex velocity

$$
u-i v=d w / d z=q e^{-i \theta},
$$

and the hodograph variable

$$
\Omega=\log \left(q_{0} / q\right)+i \theta=\tau+i \theta, \text { say. }
$$

The potential and hodograph planes are shown in figure 2. $B$ is a stagnation point because of the symmetry. The Schwarz-Christoffel transformations

$$
\begin{gathered}
w=\frac{i \Gamma}{2 \pi} \log \left[(\zeta+1)^{\frac{1}{2}}-(\zeta-1)^{\frac{1}{2}}\right]-\frac{i \Gamma}{4 \pi} \log 2, \\
\Omega=-\log \left[\{(b-1)(\zeta+1)\}^{\frac{1}{2}}-\{(b+1)(\zeta-1)\}^{\frac{1}{2}}\right]+\frac{1}{2} \log (\zeta-b)+\frac{1}{2} \log 2
\end{gathered}
$$

transform the interiors of the contours into the upper half of the $\zeta=\xi+i \eta$ plane, with $E \rightarrow \xi=-\infty, D \rightarrow \xi=-1, C \rightarrow \xi=1, B \rightarrow \xi=b$, and $A \rightarrow \xi=\infty$, where

$$
b=\left(1+R^{4}\right) / 2 R^{2} .
$$

The physical plane follows from integrating

$$
\frac{d z}{d \zeta}=\frac{1}{q_{0}} \frac{d w}{d \zeta} e^{\Omega}=-\frac{i R L}{2^{\frac{1}{2} \pi}} \frac{(\zeta-b)^{\frac{1}{2}}}{\left(\zeta^{2}-1\right)^{\frac{1}{2}}}\left[\{(b-1)(\zeta+1)\}^{\frac{1}{2}}-\{(b+1)(\zeta-1)\}^{\frac{1}{1}}\right]^{-1} .
$$

The quadrant of the vortex surface from $D$ to $C$ is mapped into the part of 


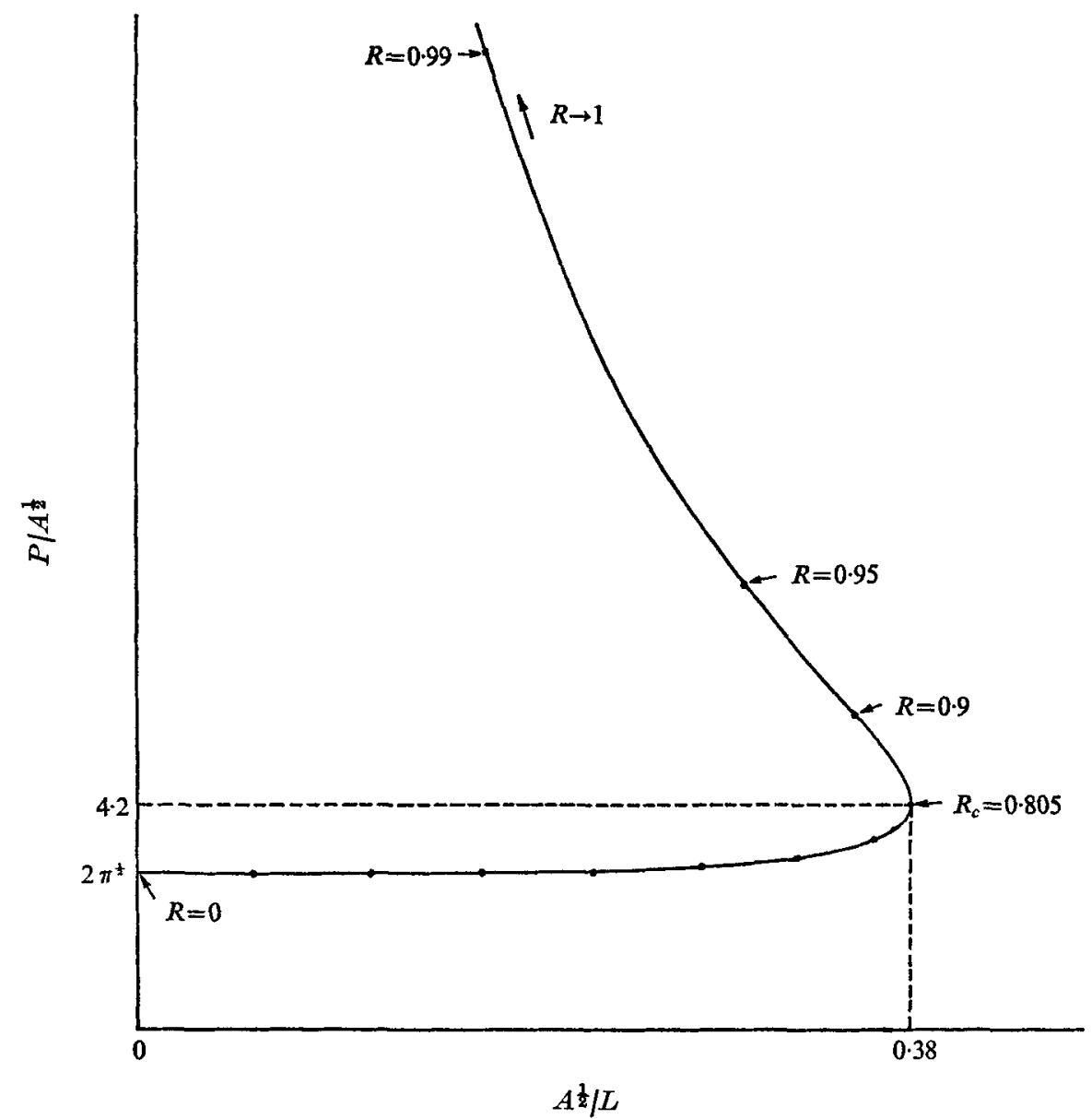

Figure 3. Perimeter length as a function of inverse distance between the cores. Variables are normalized with $A^{\frac{1}{2}}$. The seven dots on the bottom half of the curve are the values for $R=0 \cdot 1(0 \cdot 1) 0 \cdot 7$.

the real $\zeta$ axis from $\xi=-1$ to $\xi=1$. Making the substitution $\xi=-\cos 2 \lambda$, we find for the parametric equation $z=Z(\lambda)=X(\lambda)+i Y(\lambda)$ of the vortex with centre at the origin

$$
X=\frac{L}{2 \pi}\left(1+R^{2}\right) \sin ^{-1}\left(\frac{2 R \sin \lambda}{1+R^{2}}\right), \quad Y=\frac{L}{2 \pi}\left(1-R^{2}\right) \sinh ^{-1}\left(\frac{2 R \cos \lambda}{1-R^{2}}\right),
$$

where $0 \leqslant \lambda<2 \pi$ gives the complete perimeter.

The vortex is obviously circular as $R \rightarrow 0$, and flattens to the slit

$$
-\frac{1}{2} L<x<\frac{1}{2} L \quad \text { as } R \rightarrow 1 .
$$

The perimeter $P$ is $2 R L$. The area $A$ is found by numerical integration, which gives $A / L^{2}$ as a function of $R$. Figure 3 shows a plot of $P / A^{\frac{1}{2}}$ against $A^{\frac{1}{2}} / L$. Note the maximum value of $A \frac{1}{2} / L$ for a value $R=R_{c} \doteqdot 0 \cdot 805$. 


\section{Discussion}

For a given value of $A^{\frac{1}{2}} / L$, there are either two or no possible steady states. If hollow or stagnant-cored vortices of given size are placed in an array such that their separation is too small, there is no possible steady state and the vortices presumably disintegrate. For the vortex of largest area for given $L$, the length of the major axis is $0 \cdot 71 L$ and that of the minor axis is $0 \cdot 25 L$.

A similar, although not identical, behaviour holds for a single hollow vortex of area $A$ and circulation $\Gamma$ in a uniform irrotational deformation with strain rate $\epsilon$. Hill (1975) has shown that there are either one, two or no steady states according as $\epsilon A / \Gamma<0.03,0.03<\epsilon A / \Gamma<0.1$ or $0.1<\epsilon A / \Gamma$. Following Moore \& Saffman (1975), we can estimate the critical value of $A^{\frac{1}{2}} / L$ for an array from the result for a single vortex by putting $\varepsilon=\pi \Gamma / 6 L^{2}$ in the critical value for the single vortex. This gives an estimate of 0.43 for the critical value of $A \frac{1}{2} / L$. The exact value is $0 \cdot 38$, so that the approximate argument of Moore \& Saffman (1975) appears to be reasonable. The exact value of $P / A^{\frac{1}{2}}$ for the critical vortex is $4 \cdot 2$ for the array and 4.5 for the single vortex.

The existence of two possible configurations of the array suggests that at least one of them is unstable, and this should be the most deformed. We shall now verify this idea, by investigating the linear stability to infinitesimal perturbations of an array of hollow vortices, and demonstrate the existence of a class of disturbances to which the array is unstable for $R>R_{c}$ and stable for $R<R_{c}$.

\section{Stability of an array of hollow vortices}

We shall restrict attention here to infinitesimal periodic disturbances with reflexional symmetry about the centre of each vortex, which leave the centres undisplaced, because our interest lies in the stability to variations of shape. Stability of the array to disturbances which alter the positions of the vortices, i.e. of the type considered by Lamb $(1932, \S 156)$ for point vortices, is a matter for further study. (The effect of finite core size might have a bearing on the fact that Brown \& Roshko (1974) did not appear to find the Lamb-type instability.)

It is sufficient to consider the strip $-\frac{1}{2} L<x<\frac{1}{2} L, y>0$ and to use as independent co-ordinates the undisturbed velocity potential and stream function. The strip is $0<\phi<\frac{1}{2} \Gamma,-\infty<\psi<0$. A deformation of the boundary is described by the curve

$$
\psi=\delta(\phi, t), \quad 0<\phi<\frac{1}{2} \Gamma \text {. }
$$

The disturbance to the velocity potential is denoted by $\Phi(\phi, \psi, t)$. Then

$$
\begin{gathered}
\partial^{2} \Phi / \partial \phi^{2}+\partial^{2} \Phi / \partial \psi^{2}=0, \\
\Phi \rightarrow 0 \text { as } \psi \rightarrow-\infty
\end{gathered}
$$

For a hollow vortex, the pressure must be constant on its boundary; this gives a dynamic boundary condition

$$
\frac{1}{q_{0}^{2}} \frac{\partial \Phi}{\partial t}+\frac{\partial \Phi}{\partial \phi}=-\frac{2 \pi}{\Gamma} \frac{\left(b^{2}-1\right)^{\frac{1}{2}}}{b-\cos (4 \pi \phi / \Gamma)} \delta
$$


on $0<\phi<\frac{1}{2} \Gamma, \psi=0$. In addition there is a kinematic condition

$$
\frac{\partial \Phi}{\partial \psi}=\frac{1}{q_{0}^{2}} \frac{\partial \delta}{\partial t}+\frac{\partial \delta}{\partial \phi}
$$

satisfied on the undisturbed vortex.

The symmetry requires that the disturbance has period $\frac{1}{2} \Gamma$ in $\phi$. We look for normal modes of the form

$$
\begin{gathered}
\Phi=\sum_{-\infty}^{\infty} \Phi_{n} \sin \left(\frac{4 \pi n \phi}{\Gamma}+\omega t\right) \exp \left(\frac{4 \pi}{\Gamma}|n| \psi\right), \\
\frac{\delta}{b-\cos (4 \pi \phi / \Gamma)}=\sum_{-\infty}^{\infty} a_{n} \cos \left(\frac{4 \pi n \phi}{\Gamma}+\omega t\right),
\end{gathered}
$$

where $\omega$ is to be found. Inserting (5.6) and (5.7) into the boundary conditions and carrying out some straightforward algebraic manipulations, we obtain the recursion relation

$$
a_{n+1}+\left(\frac{|n| \sinh \beta}{(\sigma+n)^{2}}-2 \cosh \beta\right) a_{n}+a_{n-1}=0,
$$

for $-\infty<n<\infty$, where $b=\cosh \beta, \beta=-\log 2 R^{2}$ and $\sigma=\omega \Gamma / 4 \pi q_{0}^{2}$. The eigenvalues $\sigma$ are determined by the requirement that $a_{n} \rightarrow 0$ as $n \rightarrow \pm \infty$. If $\sigma$ is complex, the motion is unstable.

In the limit $\beta=\infty, R=0$, the eigenvalues are obviously

$$
\sigma=n \pm\left|\frac{1}{2} n\right|^{\frac{1}{2}}, \quad n= \pm 1, \pm 2, \ldots .
$$

It is easy to verify directly that these are the natural frequencies of a single hollow vortex. For $\beta$ large but not infinite, the eigenvalues can be expanded as power series in $e^{-\beta}$, and it is found that $\sigma$ remains real provided that the regular perturbation scheme remains valid.

For smaller $\beta$, numerical means need to be employed, and the method of Laplace (Jeffreys \& Jeffreys 1950, p. 486 ) is convenient. For given $\beta$, we assume a value of $\sigma$ and calculate $a_{1} / a_{0}$ and $a_{-1} / a_{0}$ as functions of $\sigma$ such that $a_{n} \rightarrow 0$ as $|n| \rightarrow+\infty$. Substitution into the recursion relation (5.8) for $n=0$ gives an equation determining $\sigma$. The details are as follows. For $n$ positive, define

$$
\alpha_{n}^{+}=\left(\frac{n+1}{n+2}\right)^{\frac{1}{2}} \frac{a_{n+1}}{a_{n}} e^{\beta}-1 .
$$

It can be shown that $\alpha_{n}^{+}=O\left(n^{-2}\right)$ for large $n$ when $a_{n}$ decays as $n \rightarrow \infty$. From the recursion relation,

$$
\alpha_{n-1}^{+}=-1-e^{\beta}\left[e^{-\beta}\left(1+\frac{2}{n}\right)^{\frac{1}{2}}\left(1+\alpha_{n}^{+}\right)-\left(1+\frac{1}{n}\right)^{\frac{1}{2}}\left(2 \cosh \beta-\frac{n}{(\sigma+n)^{2}} \sinh \beta\right)\right]^{-1} .
$$

The asymptotic behaviour of $\alpha_{n}^{+}$gives a starting value from which $\alpha_{0}^{+}(\sigma, \beta)$ can be calculated numerically. We proceed similarly for the $a_{-n}(n>0)$, defining

$$
\alpha_{n}^{-}=\left(\frac{n+1}{n+2}\right)^{\frac{1}{2}} \frac{a_{-n-1}}{a_{-n}} e^{\beta}-1
$$


and calculating $\alpha_{0}^{-}(\sigma, \beta)$. Because of the symmetry of the recursion relation

$$
\alpha_{0}^{-}(\sigma, \beta)=\alpha_{0}^{+}(-\sigma, \beta) .
$$

The recursion relation for $n=0$ gives

$$
f\left(\sigma^{2}, \beta\right) \equiv \alpha_{0}^{+}(\sigma, \beta)+\alpha_{0}^{-}(\sigma, \beta)=2^{\frac{1}{2}} e^{\beta} \cosh \beta-2 .
$$

Since the left-hand side can be found numerically as a function of $\sigma$, the roots of (5.14) are obtained in a straightforward manner as functions of $\beta$.

Note that the roots occur in pairs, $\pm \sigma$. The roots are known for large $\beta$, so the procedure is to follow the roots numerically as $\beta$ decreases. The smallest positive root $\sigma_{1}(\beta)$, say, turns out to be the one of interest. As $\beta$ decreases, $\sigma_{1}$ decreases from $1-1 / 2^{\frac{1}{2}}$ at $\beta=\infty$ to zero at $\beta=0 \cdot 434$. This value can be found analytically as the recursion relation can be solved in closed form (using generating functions) when $\sigma=0$. For $\beta$ less than $0 \cdot 434$, equation (5.14) is found to have roots with $\sigma^{2}<0$, demonstrating that there is an exchange of stabilities. It can be shown that the other roots remain real.

The critical value of $\beta$ at which the array becomes unstable to disturbances of the type considered here gives the same value of $R, 0 \cdot 805$, as that at which $A^{\frac{1}{2}} / L$ is a maximum, thereby demonstrating that, when there are two possible configurations, the more deformed is unstable to disturbances for which the less deformed is stable (cf. Moore \& Saffman 1971).

This work was supported by the U.S. Army Research Office, Durham, under contract DAHC 04-75-C-0009.

\section{Appendix}

Consider a member of the linear array of hollow or stagnant vortices with the geometry as shown in figure 4 . We assume periodicity of the array and reflexional symmetry only.

Using the hodograph variable defined by (3.2) we then require that $\tau$ satisfies Laplace's equation in the strip $A B C D E F$ and the following boundary conditions:

$$
\begin{aligned}
& \tau=0 \text { along } C D, \\
& \tau \text { has period } \frac{1}{2} \Gamma \text { in } \phi, \\
& \tau \sim-\log R \text { as } \psi \rightarrow-\infty
\end{aligned}
$$

Moreover, we want $\tau$ to have the correct behaviour at the stagnation points $B$ and $E$. Noting that $d w / d z \sim\left(w-w_{0}\right)^{\frac{1}{2}}$ at a stagnation point, we can separate out the singular behaviour of $\tau$ at such points by using functions which behave locally as required. Including terms ensuring correct asymptotic behaviour and without violating (A 2), we have

$$
\begin{aligned}
\tau=\operatorname{Re}\left\{-\log 2 R-\frac{1}{2} \log \left(\cos \frac{2 \pi}{\Gamma} w+i \sinh \frac{2 \pi}{\Gamma} \psi_{0}\right)\right. \\
\left.-\frac{1}{2} \log \left(\cos \frac{2 \pi}{\Gamma} w-i \sinh \frac{2 \pi}{\Gamma} \psi_{0}\right)-\frac{2 \pi}{\Gamma} i w\right\}+H(\phi, \psi),
\end{aligned}
$$



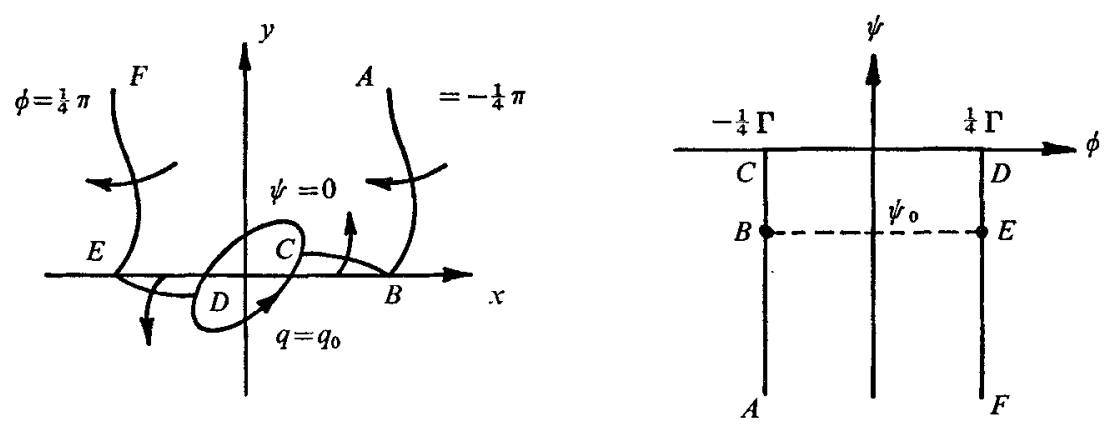

Fiaure 4. Physical and potential planes for an array with reflexional symmetry but not necessarily fore-and-aft symmetry. $\psi_{0}$ is the value of the stream function at the stagnation points $B$ and $E$.

where $H$ satisfies Laplace's equation and (A2), and is bounded on the strip. Clearly

$$
H=\sum_{n=0}^{\infty} \exp (4 \pi n \psi / \Gamma)\left(A_{n} \sin \frac{4 \pi n \phi}{\Gamma}+B_{n} \cos \frac{4 \pi n \phi}{\Gamma}\right) .
$$

Now for $\tau$ to satisfy (A 1) we require $A_{n}=0$, and hence there is fore-and-aft symmetry. Further,

$$
0=-\log 2 R-\frac{1}{2} \log \left(\cos ^{2} \frac{2 \pi \phi}{\Gamma}+\sinh ^{2} \frac{2 \pi \psi_{0}}{\Gamma}\right)+\sum_{n=0}^{\infty} B_{n} \cos \frac{4 \pi n \phi}{\Gamma},
$$

so that the $B_{n}$ are all uniquely determined. Note that for the correct asymptotic behaviour we require $B_{0}=0$, and so

$$
\log 2 R+\frac{1}{\Gamma} \int_{-\frac{1}{4} \Gamma}^{\frac{1}{4} \Gamma} \log \left(\cos ^{2} \frac{2 \pi \phi}{\Gamma}+\sinh ^{2} \frac{2 \pi \psi_{0}}{\Gamma}\right) d \phi=0 .
$$

Writing $b=1+2 \sinh ^{2}\left(2 \pi \psi_{0} / \Gamma\right)$, we find that (A 4$)$ implies

$$
b+\left(b^{2}-1\right)^{\frac{1}{2}}=1 / R^{2} .
$$

Since $b>1$, then $R<1$.

\section{REFERENCES}

Brown, G. L. \& Roshko, A. 1974 On density effects and large structure in turbulent mixing layers. J. Fluid Mech. 64, 775 .

Hrux, F. M. 1975 Ph.D. thesis, Imperial College, London.

JEFFREYS, H. \& JEFFREYs, B. S. 1950 Methods of Mathematical Physics. Cambridge University Press.

Lamb, H. 1932 Hydrodynamics. Cambridge University Press.

Moore, D. W. \& Safrmar, P. G. 1971 Structure of a line vortex in an imposed strain. In Aircraft Wake Turbulence and its Detection (ed. Olsen, Goldberg \& Rogers), p. 339. Plenum.

Moore, D. W. \& Saffman, P. G. 1975 The density of organized vortices in a turbulent mixing layer. J. Fluid Mech. 69, 465. 